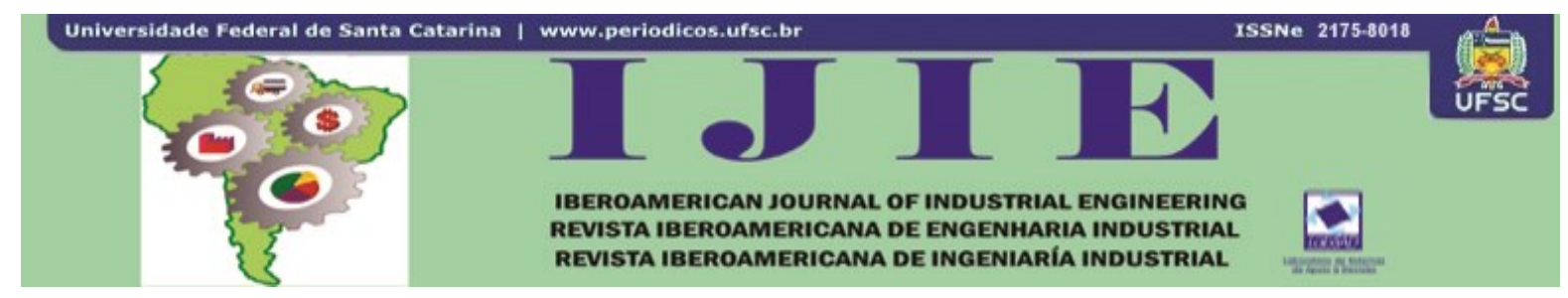

\title{
PLANEAMIENTO OPERATIVO DE REDES DE EVAPORACIÓN
}

\author{
Humberto Heluane ${ }^{1}$ \\ Aníbal M. Blanco ${ }^{2}$ \\ María Rosa Hernández ${ }^{3}$ \\ José Alberto Bandoni ${ }^{4}$
}

RESUMEN: La evaporación es una operación crítica para un manejo eficiente del agua y la energía en ciertos procesos industriales. En este trabajo se aborda el planeamiento operativo de sistemas de evaporación de múltiple efecto. El objetivo del estudio es determinar la secuencia de paradas de las líneas de evaporación para realizar la limpieza de los equipos. Se presenta un modelo no lineal mezcla entera basado en una representación discreta del tiempo que tiene en cuenta la pérdida de eficiencia por ensuciamiento con el uso. El modelo se aplica al sector de evaporación de una planta de producción de azúcar de caña obteniéndose la secuencia de paradas, los tiempos de operación de las líneas y los perfiles de concentración y caudal alimentado a cada una de ellas. La optimización de la operación del sistema de evaporación del caso de estudio, permite obtener una mayor concentración del producto de la evaporación con el consecuente ahorro de energía del arreglo evaporación/cristalización.

Palabras claves: Evaporación. Planeamiento operativo. Programación matemática. Azúcar de caña.

\section{INTRODUCCIÓN}

Los sistemas de evaporación, de importancia fundamental en muchos procesos industriales, consisten en series de evaporadores que operan en paralelo (Figura 1). Estos sistemas están sujetos al ensuciamiento de las superficies de transferencia de calor, en especial cuando están involucrados fluidos que contienen altas concentraciones de materia disuelta o en suspensión.

Este ensuciamiento genera un incremento en la resistencia a la transferencia de calor con el consecuente incremento en el consumo de energía, mano de obra para limpieza y

\footnotetext{
1 Profesor Adjunto, Departamento de Ingeniería de Procesos y Gestión Industrial, FACET - Universidad Nacional de Tucumán, Av. Independência 1800, (4000) -Tucumán, Argentina. E-mail: hheluane@herrera.unt.edu.ar.

${ }^{2}$ Doctor in Chemical Engineering, PLAPIQUI (UNS - CONICET), Camino La Carrindanga km. 7 (8000) Bahía Blanca/Argentina. E-mail: ablanco@plapiqui.edu.ar.

${ }^{3}$ Mag. Departamento de Ingeniería de Procesos y Gestión Industrial, FACET - Universidad Nacional de Tucumán, Av. Independência 1800, (4000) -Tucumán/Argentina. E-mail: rhernandez@,herrera.unt.edu.ar.

4 Profesor Titular (DE), Doctor en Ingeniería Química PLAPIQUI (UNS - CONICET), Camino La Carrindanga km. 7 (8000) - Bahía Blanca/Argentina. E-mail: abandoni@plapiqui.edu.ar.
} 
pérdida de oportunidades de producción. Como consecuencia, es necesario detener frecuentemente los distintos trenes para su limpieza con el objetivo de restablecer la eficiencia original. Esta situación da lugar a un problema de planeamiento operativo óptimo de la red de evaporación.

La programación matemática se ha utilizado anteriormente en el planeamiento de operaciones de corto y largo plazo en procesos continuos y discontinuos cuyos desempeños decaen en el tiempo. Georgiadis, Papageorgiou y Macchietto (1999), Georgiadis y Papageorgiou (2000) han utilizado modelos de ensuciamiento en problemas de mantenimiento de redes de intercambio de calor. El planeamiento de redes de evaporadores en paralelo fue abordada por Heluane et al. (2004, 2007). Finalmente, varios autores estudiaron el planeamiento de otros procesos con pérdida de eficiencia (JAIN; GROSSMANN, 1998; HOUZE; JUHASZ; GROSSMANN, 2003; SCHULZ; BANDONI; DIAZ, 2006).

En este trabajo se presenta un modelo de optimización multi-período que permite planear la operación de sistemas de evaporación. El criterio de desempeño adoptado está asociado a la minimización del consumo de vapor asegurando simultáneamente una concentración determinada de la solución de salida.

En general las paradas de los trenes de evaporación se planean de manera cíclica para que puedan ser repetidos en el tiempo. El modelo resultante es no lineal mezcla entera (MINLP) y se utilizó como caso de estudio el sector de evaporación de un ingenio azucarero.

Este trabajo está estructurado de la siguiente manera: Primero se presenta el modelo multiperíodo donde, además de los balances de conservación, se detallan las variables binarias que se introducen para determinar la secuencia de paradas para efectuar la limpieza/mantenimiento de las líneas de evaporación. A continuación se presenta como caso de estudio el subsistema de evaporación del proceso de obtención de azúcar. Se compara los resultados de la operación habitual de dicho subsistema con los obtenidos al implementar las variables de diseño optimizadas con el modelo presentado en este trabajo.

\section{MODELO MINLP}

En una red de evaporación de múltiple efecto, varias líneas de evaporación de múltiple efecto operan en paralelo (Figura 1). Cada equipo en la línea trabaja a una presión progresivamente inferior. La energía requerida para lograr la ebullición en la primera unidad esta proporcionada por vapor de caldera. 
El vapor generado por la primera unidad se utiliza para calentar la segunda. El vapor generado en la segunda unidad se emplea para calentar la siguiente y así sucesivamente. Cualquier vapor puede utilizarse como fuente de energía en las unidades aguas abajo debido a que su temperatura es superior al punto de ebullición del líquido de cualquiera de esos equipos.

La formulación matemática del modelo de optimización adopta las siguientes hipótesis para la operación de los evaporadores:

a) El sistema opera en estado pseudo-estacionario y sin pérdida de masa

b) Pérdidas de energía despreciables en los equipos

c) Se considera despreciable el incremento del punto de ebullición en los evaporadores.

d) Se asumen condiciones estables dentro de cada unidad de evaporación.

e) La planta dispone un sistema de control capaz de distribuir los caudales óptimos a las diferentes líneas de evaporación.

El problema considerado en este trabajo consiste en determinar cuánto caudal hay que alimentar a cada línea de evaporación en cada instante de tiempo, en qué momento debe detenerse la operación de cada una de las líneas y en qué secuencia, y como son los perfiles de caudal y concentración a lo largo de las líneas.

Cabe aclarar que dado que red de equipos está disponible, la función objetivo a optimizar corresponde únicamente al costo operativo del sistema. Dicha función dependerá en general del problema bajo estudio y será desarrollada más adelante para la aplicación específica de este trabajo.

Se adopta un horizonte de planeamiento de 14 días con una representación discreta del tiempo tomando como base 12 horas, que es el tiempo requerido para realizar la limpieza de una línea. Se impone la realización de dos limpiezas en el horizonte considerado con el objeto de representar la práctica industrial de una limpieza por semana de cada línea. 


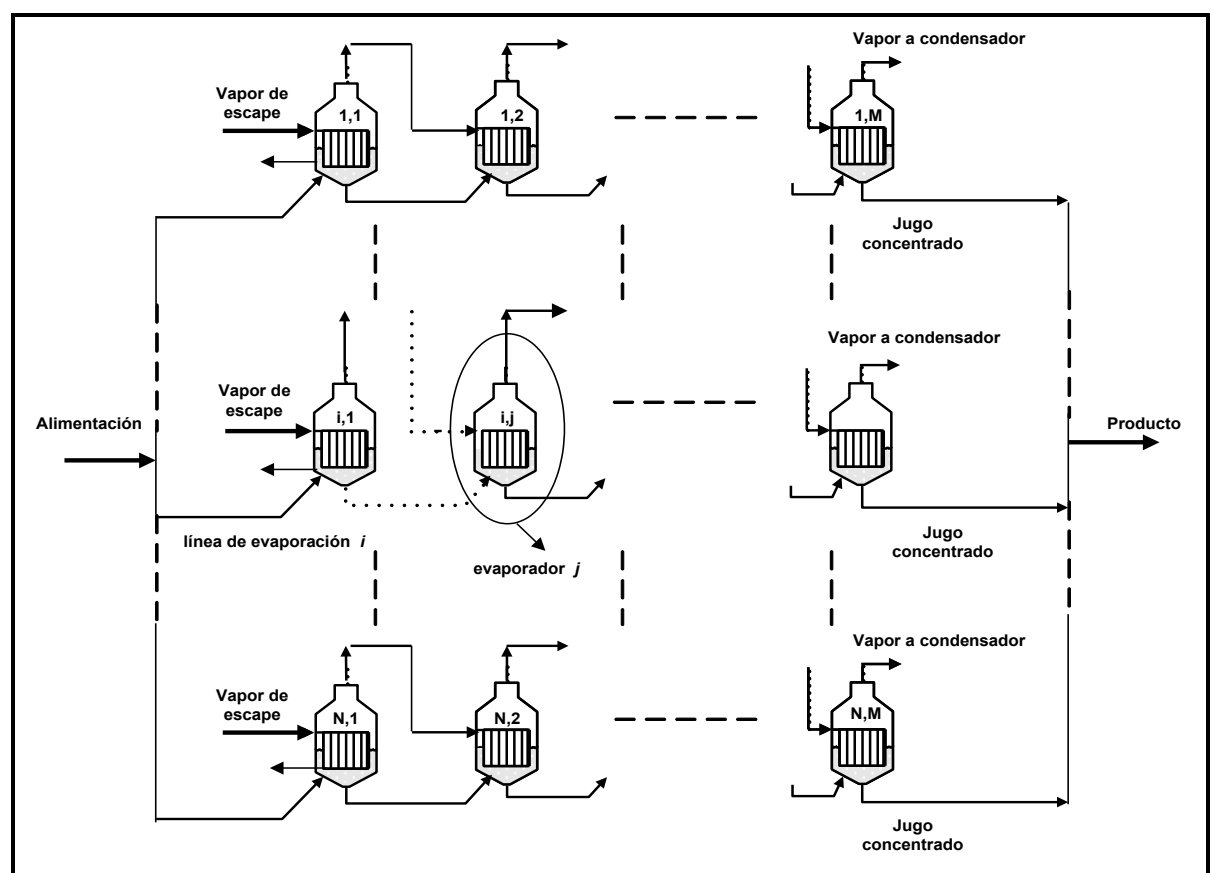

Figura 1 - Esquema genérico de una red de evaporadores de efecto múltiple

Uno de los objetivos del modelo es determinar en qué momento debe detenerse una línea para el mantenimiento/limpieza de los evaporadores. Debido a los depósitos de materia en la superficie de transferencia, la resistencia global a la transferencia de calor aumenta en el tiempo mientras está operando el equipo.

En este trabajo se adoptó una dependencia lineal de la resistencia global a la transferencia de calor con el tiempo, basado en cálculos estadísticos de datos obtenidos en planta. Para el modelado de las paradas por limpieza de las líneas de evaporación, se utilizaron variables binarias de acuerdo a la lógica propuesta por Houze, Juhasz y Grossmann (2003).

La Ecuación 1 se utiliza para modelar la perdida de eficiencia de las unidades en el tiempo debido a ensuciamiento. $R_{i, j, t}$ representa la resistencia global a la transferencia de calor para el equipo $(i, j)$ en el período $t$.

$$
R_{i, j, t}=C 1_{i, j}+C 2_{i, j} t
$$

donde $C 1_{i, j}$ es la resistencia global a la transferencia de calor inicial para la unidad $(i, j)$ y $C 2_{i, j}$ es la velocidad de ensuciamiento.

Para modelar las paradas para las limpiezas, y representar el comportamiento de la resistencia global de transferencia de calor en los evaporadores se utilizan dos conjuntos de variables binarias, $z_{h, i, t}$ e $y_{m, i, t}$, cuyas definiciones se muestran en la Figura 2 y se describen a continuación. 


$$
\begin{aligned}
& y_{1, i, t} \begin{cases}=1 & \text { cuando la línea } i \text { realiza la primera limpieza en el período } t \\
=0 & \text { en cualquier otro período }\end{cases} \\
& y_{2, i, t} \begin{cases}=1 & \text { cuando la línea } i \text { realiza la segunda limpieza en el período } t \\
=0 & \text { en cualquier otro período }\end{cases} \\
& y_{3, i, t} \begin{cases}=1 & \text { cuando la línea } i \text { realiza las primera y segunda limpiezas en } \\
=0 & \text { en correspond ientes períodos } t\end{cases}
\end{aligned}
$$$$
z_{1, i, t} \begin{cases}=1 & \text { desde el inicio de la operación de la línea i hasta finalizar la primera } \\ =0 & \text { en cualquier otro período }\end{cases}
$$$$
z_{2, i, t} \begin{cases}=1 & \text { desde la finalizaci ón la primera limpieza hasta la finalizaci ón la segunda } \\ =0 & \text { en cualquier otro período }\end{cases}
$$$$
z_{3, i, t} \begin{cases}=1 & \text { desde la finalizaci ón la segunda limpieza de la línea } i \text { hasta el último período } \\ =0 & \text { en cualquier otro período }\end{cases}
$$

Definición de las variables binarias $z_{h, i, t}$ : Las Ecuaciones 12 a 24 definen a las variables $z_{h, i, t}$, donde $T P_{k, i}$ es el período de tiempo $k$ en que debe detenerse la línea de evaporación $i$ para realizar tareas de limpieza. En este modelo se adoptan dos limpiezas en el horizonte de tiempo, por lo que el subíndice $k$ puede tomar el valor 1 ó 2 .

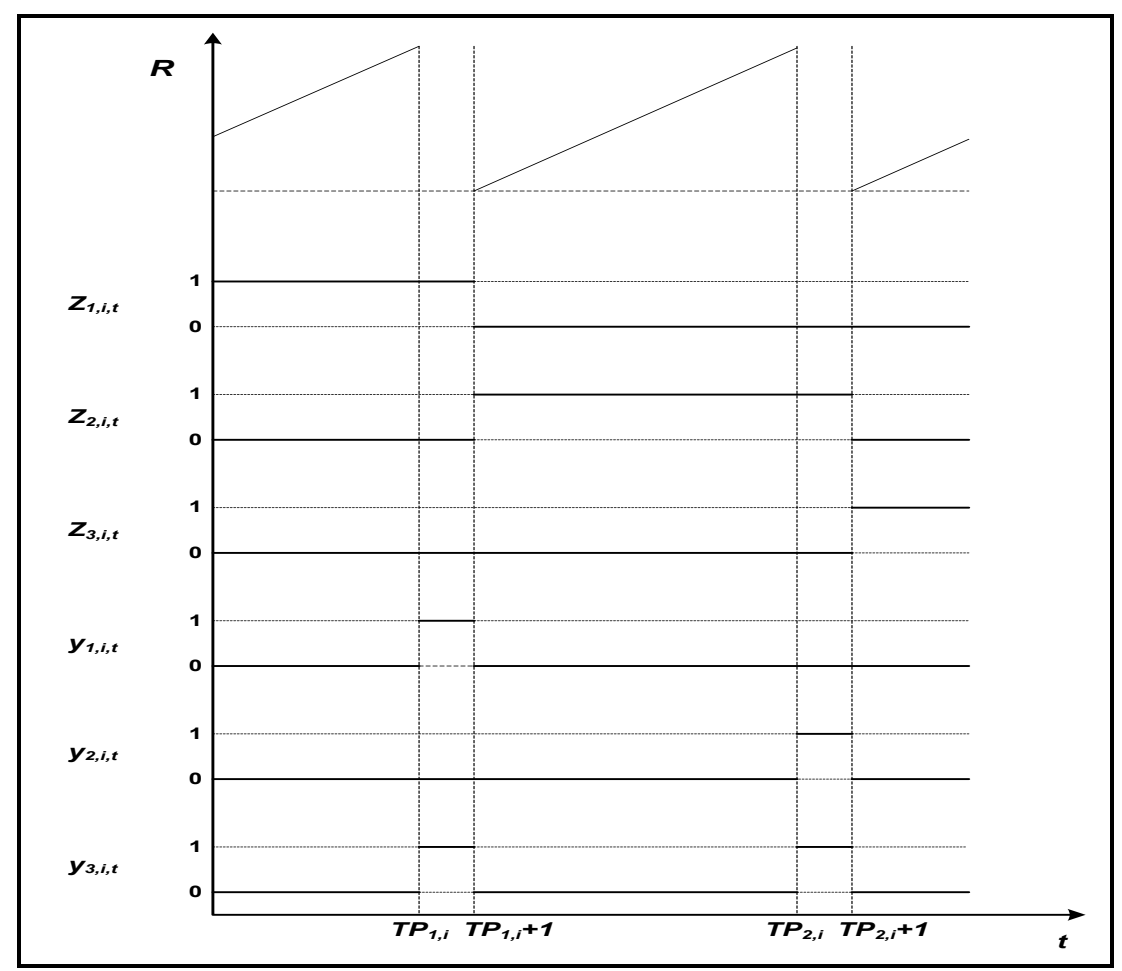

Figura 2 - Representación de las variables binarias $z_{h, i, t}$ e $y_{m, i, t}$ Fuente: Obtenidos del modelo propuesto 
Las Ecuaciones 2 a 9 definen a las variables $z_{h, i, t}$, donde $B M$ es la constante $b i g M$. Se muestran a continuación las ecuaciones que definen solo dos limpiezas, pero pueden ser extendías para cualquier número de limpiezas. Para la primera limpieza y si el período $t$ es menor o igual a $\left(T C_{1, i}+1\right)$ entonces $z_{1, i, t}$ es igual a 1 ; de otro modo, es igual a 0 .

$$
\begin{array}{ll}
t \leq T C_{1, i}+1+B M 1\left(1-z_{1, i, t}\right) & \forall i, t \\
t \geq T C_{1, i}+2-B M 1 z_{1, i, t} & \forall i, t
\end{array}
$$

La variable binaria $z_{2, i, t}$ toma el valor 1 para el intervalo de tiempo entre la primera y segunda limpiezas para una línea de evaporación $i$, es decir cuando, $\left(T C_{1, i}+2\right) \leq t \leq\left(T C_{2, i}\right.$ +1 ); para cualquier otro tiempo esta variable binaria es igual a 0 .

$$
\begin{array}{lr}
t \geq T C_{1, i}+2-B M 1\left(1-z_{2, i, t}\right) & \forall i, t \\
t \leq T C_{1, i}+1+B M 1 a_{i, t} & \forall i, t \\
t \geq T C_{1, i}+2-B M 1\left(1-a_{i, t}\right) & \forall i, t \\
t \leq T C_{2, i}+1+B M 1\left(1-z_{2, i, t}\right) & \forall i, t \\
t \leq T C_{2, i}+1+B M 1\left(1-b_{i, t}\right) & \forall i, t \\
t \geq T C_{2, i}+2-B M 1 b_{i, t} & \forall i, t
\end{array}
$$

Para poder definir $z_{2, i, t}$ es necesario introducir las variable binarias auxiliares $a_{i, t}$ y $b_{i, t}$ que se comentan a continuación: si $a_{i, t}=1$ y $b_{i, t}=1$ entonces $z_{2, i, t}=1$. Cuando ya sea $a_{i, t}=0 \mathrm{o}$ $b_{i, t}=0$ entonces $z_{2, i, t}=0$ (Ecuación 10).

Para cualquier período $t$, cada línea de evaporación sólo puede estar en una etapa $\mathrm{h}$ (primera, segunda o tercera etapa).

La primera etapa $(h=1)$ corresponde a los períodos en los cuales $z_{1, i, t}=1$.

La segunda etapa $(h=2)$ corresponde a los períodos en los cuales $z_{2, i, t}=1$.

La tercera etapa $(h=3)$ corresponde a los períodos en los cuales $\mathrm{z}_{3, i, t}=1$.

$$
\sum_{h=1}^{3} z_{h, i, t}=1 \quad \forall i, t
$$

Definición de las variables binarias $y_{m, i, t}$ : El segundo grupo de variables binarias $y_{m, i, t}$ se utiliza para definir $T_{P k, i}$ mediante la Ecuación 11 y para hacer que las variables continuas asociadas a cada línea de evaporación se igualen a cero durante los períodos de limpieza. La 
variable $y_{m, i, t}$ es igual a 1 , en el período $t$ cuando la línea $i$ está detenida por tareas de limpieza, en cualquier otro período toma el valor cero (ver Figura 2).

Las variables binarias $y_{1, i, t}$ se definen de la siguiente manera: como se explicó anteriormente $y_{1, i, t}=1$ en el período $T P_{1, i}$ correspondiente a la primera limpieza de la línea de evaporación $i$.

$$
T P_{1, i}=\sum_{t}(t-1) y_{1, i, t}
$$

Las variables binarias $y_{2, i, t}$ se definen de la siguiente manera: nuevamente, como se explicó anteriormente $y_{2, i, t}=1$ en el período $T P_{1, i}$ correspondiente a la segunda limpieza de la línea de evaporación $i$.

$$
T P_{2, i}=\sum_{t}(t-1) y_{2, i, t} \quad \forall i
$$

Las variables binarias $y_{3, i, t}$ se definen de la siguiente manera: se explicó antes que la variable $y_{3, i, t}=1$ cuando se realizan la primera o segunda limpieza de la línea $i$, es decir, $y_{3, i, t}$ toma el valor 1 cuando $y_{1, i, t}$ ó $y_{2, i, t}$ toman el valor 1 . La variable $y_{3, i, t}$ toma el valor cero solamente cuando ambas variables $y_{1, i, t}$ e $y_{2, i, t}$ toman el valor cero. Las expresiones matemáticas de estas proposiciones son las desigualdades (Ecuaciones 13 a 15).

$$
\begin{array}{lc}
y_{3, i, t}-y_{1, i, t} \geq 0 & \forall i, t \\
y_{3, i, t}-y_{2, i, t} \geq 0 & \forall i, t \\
y_{1, i, t}+y_{2, i, t}-y_{3, i, t} \geq 0 & \forall i, t
\end{array}
$$

Determinación del número de limpiezas: Para considerar que existan dos limpiezas en el horizonte de tiempo se agrega las siguientes restricciones (Ecuaciones 16 y 17):

$$
\begin{array}{ll}
\sum_{t} y_{1, l, t}=1 & \forall l, t \\
\sum_{t} y_{2, l, t}=1 & \forall l, t
\end{array}
$$

Estado de la limpieza de la línea: Cada línea de evaporación solamente puede estar en la primera o en la segunda limpieza, y para ello se agrega las desigualdades (Ecuación 18) como restricción del modelo. 


$$
\sum_{m=1}^{2} y_{m, i, t} \leq 1
$$

Única línea limpiando por vez:

Por razones de operación, se considera que solamente se puede limpiar una línea por vez, y esto se indica mediante las siguientes desigualdades.

$$
\sum_{i=1}^{N} \sum_{m=1}^{2} y_{m, i, t} \leq 1 \quad \forall t
$$

Representación de la resistencia global a la transferencia de calor: Las siguientes inecuaciones representan el comportamiento de la resistencia global a la transferencia de calor para los diferentes períodos $t$. Para períodos anteriores o iguales a $T C_{l, i}$, los valores de $R_{i, j, t}$ quedan determinados por las Ecuaciones 20 y 21 :

$$
\begin{array}{r}
R_{i, j, t} \geq C 1_{i, j}+C 2_{i, j} t-B M 1\left(1-z_{1, i, t}\right) \quad \forall i, j, t \\
R_{i, j, t} \leq C 1_{i, j}+C 2_{i, j} t+B M 1\left(1-z_{1, i, t}\right) \quad \forall i, j, t
\end{array}
$$

Para los períodos entre la primera y segunda limpiezas, $\left(T C_{1, i}+1\right) \leq t \leq T C_{2, i}$, los valores de $R_{i, j, t}$ se calculan mediante las Ecuaciones 22 y 23 , donde $C 1_{i, j}=R_{i, j}^{0}$ para los períodos $t$ correspondientes ya que el equipo comienza a operar limpio.

$$
\begin{array}{lr}
R_{i, j, t} \geq R_{i, j}^{0}+C 2_{i, j}\left[t-\left(T C_{1, i}+1\right)\right]-B M 1\left(1-z_{2, i, t}\right) & \forall i, j, t \\
R_{i, j, t} \leq R_{i, j}^{0}+C 2_{i, j}\left[t-\left(T C_{1, i}+1\right)\right]+B M 1\left(1-z_{2, i, t}\right) & \forall i, j, t
\end{array}
$$

Finalmente, para la tercera etapa del modelo, cuando $t \geq T P_{2, i}+1$, los valores de $R_{i, j, t}$ se calculan mediante las Ecuaciones 24 y 25 . En esta tercera etapa $z_{3, i, t}=1$, por lo tanto la resistencia global se calcula mediante la Ecuación 1 para los períodos $t$ correspondientes con $C l_{i, j}=R_{i, j}^{0}$ para los períodos $t$ correspondientes ya que el equipo comienza a operar limpio, mientras que para cualquier otra etapa, $z_{3, i, t}=0$ y las desigualdades (Ecuaciones 24 y 25) se relajan.

$$
R_{i, j, t} \geq R_{i, j}^{0}+C 2_{i, j} \cdot\left[t-\left(T P_{2, i}+1\right)\right]-B M 1 \cdot\left(1-z_{3, i, t}\right) \quad \forall i, j, t
$$




$$
R_{i, j, t} \leq R_{i, j}^{0}+C 2_{i, j} \cdot\left[t-\left(T P_{2, i}+1\right)\right]+B M 1 \cdot\left(1-z_{3, i, t}\right) \quad \forall i, j, t
$$

Balances de masa y energía: Mediante las siguientes inecuaciones se logra que los flujos de masa y las concentraciones se anulen en los periodos en los que se realiza la limpieza de la línea $i$.

$$
\begin{array}{rr}
F I_{i, t} \leq F_{i}^{u p} \cdot\left(1-y_{3, i, t}\right) & \forall i, t \\
F I_{i, t} \geq F_{i}^{l o} \cdot\left(1-y_{3, i, t}\right) & \forall i, t \\
F_{i, j, t} \leq F_{i}^{u p} \cdot\left(1-y_{3, i, t}\right) & \forall i, t, j=1,2,3,4 \\
F_{i, j, t} \geq F_{i}^{l o} \cdot\left(1-y_{3, i, t}\right) & \forall i, t, j=1,2,3,4
\end{array}
$$

Donde $F^{u p}$ es el caudal de alimentación máximo y $F^{l o}{ }_{i}$ el caudal mínimo admitido por la línea $i$. El caudal mínimo y máximo admitido por una línea dependerá del tamaño de los equipos utilizados en ella. Cuando una línea $i$ está operando $\left(y_{3, i, t}=0\right)$, el vapor generado en las unidades de evaporación pueden ser calculados de los balances de energía, como se muestra a continuación (Ecuaciones 30 y 31).

$$
\begin{array}{ll}
V_{i, j, t} \geq \frac{A_{i, j} \cdot \Delta \theta_{i, j}}{\lambda_{i, j}} \frac{1}{R_{i, j, t}}-B M 1 \cdot y_{3, i, t} & \forall i, j, t \\
V_{i, j, t} \leq \frac{A_{i, j} \cdot \Delta \theta_{i, j}}{\lambda_{i, j}} \frac{1}{R_{i, j, t}}+B M 1 \cdot y_{3, i, t} & \forall i, j, t
\end{array}
$$

Los balances de masa para las primeras unidades de las líneas de evaporación están dados por la Ecuación 32 y para las subsiguientes unidades de las líneas de evaporación están dados por las Ecuación 33.

$$
\begin{array}{cc}
x_{0} \cdot F I_{i, t}=x_{i, j, t} \cdot F_{i, j, t} & \forall i, t, j=1 \\
x_{i, j-1, t} \cdot F_{i, j-1, t}=x_{i, j, t} \cdot F_{i, j, t} & \forall i, t, j=2,3, \cdots M
\end{array}
$$

Los balances totales para las primeras unidades de las líneas de evaporación están dados por las Ecuación 34 y los balances totales para las subsiguientes unidades de las líneas de evaporación están dados por las Ecuación 35.

$$
F I_{i, t}=F_{i, j, t}+V_{i, j, t} \quad \forall i, t, j=1
$$




$$
F_{i, j-1, t}=F_{i, j, t}+V_{i, j, t}
$$

La Ecuación 36 asegura que todo el caudal que ingresa al sistema sea procesado.

$$
F M=\sum_{i=1}^{N} F I_{i, t} \quad \forall t
$$

El modelo desarrollado contempla líneas de evaporación trabajando en paralelo y como resultado se obtiene la secuencia de paradas, los tiempos de operación de las líneas y los perfiles de concentración y caudal alimentado a cada una de ellas. El modelo fue presentado para dos paradas pero puede ser extendido a $n$ paradas con expresiones análogas a las Ecuaciones (2) a (9).

En este trabajo se utilizó una expresión lineal (Ecuación 1) para reflejar el aumento de la resistencia global a la transferencia de calor $R$. Esta correlación se obtuvo a partir de datos experimentales de la variación de $R$ en el tiempo utilizando el método de regresión lineal (HELUANE, 2010). En caso que la variación de $R$ en el tiempo presente otro perfil, el modelo de optimización presentado puede ser utilizado reemplazando en la Ecuación 1 la expresión que corresponda.

\section{CASO DE ESTUDIO}

La formulación presentada anteriormente puede ser aplicada a cualquier sistema de evaporación. Como caso de estudio se tomó el sector de evaporación de un ingenio azucarero. La evaporación en la industria azucarera se utiliza para concentrar el jugo clarificado antes de ingresar a la etapa de cristalización.

El objetivo de ambas operaciones es eliminar agua del jugo para obtener cristales de sacarosa. En estas plantas el ensuciamiento es generalmente severo dada la naturaleza viscosa del fluido que circula por los evaporadores y por lo tanto resulta una aplicación interesante para el modelo propuesto.

El proceso de evaporación es más eficiente que el de cristalización desde el punto de vista energético debido al arreglo múltiple-efecto. En una línea múltiple-efecto con $\mathrm{M}$ evaporadores, el agua extraída es aproximadamente $M$ veces el vapor inyectado al primer evaporador.

Por otro lado, en la cristalización, la cantidad de agua extraída es aproximadamente igual al vapor consumido. Por lo tanto es necesario considerar simultáneamente ambas operaciones, evaporación y cristalización, para evaluar los costos operativos del proceso. 
Como los costos de limpieza son despreciables frente a los del vapor consumido, la función objetivo a minimizar serán los del vapor consumido en las etapas de evaporación y cristalización.

Como se demostró en Heluane et al. (2004), el costo de operación mínimo se obtendrá maximizando la concentración a la salida de la evaporación, para una dada configuración de la red de evaporación.

La función objetivo utilizada es la concentración a la salida de las líneas de evaporación, ya que esto implica menor consumo de vapor de caldera y por lo tanto menores costos de operación. La función objetivo está dada por la Ecuación 37.

$$
F O=\sum_{t} x_{i, M, t}
$$

Donde $x_{i, M, t}$ es la concentración en la última unidad $M$ de evaporación de la línea $i$ para el período $t$.

El caso corresponde a un ingenio azucarero de la provincia de Tucumán (Argentina) donde el subsistema de evaporación consiste en 5 líneas con 5 efectos cada una que pueden operar simultáneamente, donde cada una de ellas se detiene para limpieza/mantenimiento una vez por semana, en días diferentes, necesitándose para ello 12 horas de trabajo por línea.

Cuando alguna de las líneas se detiene, el caudal correspondiente es repartido uniformemente en las 4 líneas que quedan funcionando. La ubicación y el tamaño de cada equipo en las líneas se muestran en la Tabla 1. Los datos reales de planta fueron obtenidos por comunicación personal por parte de los técnicos responsables de la operación de la subsección de evaporación del ingenio.

\section{RESULTADOS}

El caso de estudio es una red con 5 líneas de evaporación. Cada línea es un sistema múltiple con 5 efectos. Se desea planear la operación de dicha red para procesar $800 \mathrm{t} / \mathrm{h}$ de jugo clarificado de una concentración de azúcar igual a 16 t azúcar /t de jugo.

Por requerimientos operativos, en ningún caso la concentración del jugo durante la evaporación debe superar los 70 t azúcar /t de jugo. El tiempo de limpieza para cada línea es de 12 horas, coincidente con un periodo del horizonte de planeamiento discreteado.

El vapor latente de vaporización se toma para todos los casos igual al del agua (530 $\mathrm{kcal} / \mathrm{kg})$. El caudal máximo $\left(F_{i}^{u p}\right)$ impuesto para la alimentación a cada línea fue $450 \mathrm{t} / \mathrm{h}$. La dimensión de cada evaporador de la red se muestra en la Tabla 1. 
Tabela 1 - Áreas de intercambio $\left(\mathrm{m}^{2}\right)$ de los evaporadores de la red

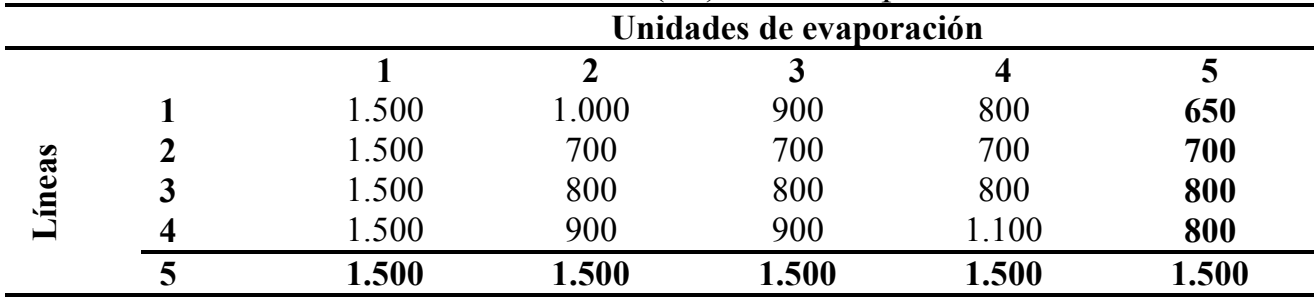

Fuente: Basados en un ingenio azucarero de la provincia de Tucumán (Argentina)

El problema resultante consiste de 11.986 restricciones, 4.155 variables continuas y 1.120 variables binarias. El caso fue implementado en GAMS (2008a) y resuelto usando DICOPT++ con CPLEX y CONOPT3 (2008b), en una PC Pentium 4 de 512 Mb de RAM. El valor óptimo de la función objetivo resultó ser 5717. Los caudales óptimos alimentados a cada línea que se obtienen como resultado de la optimización se muestran en la Figura 3.

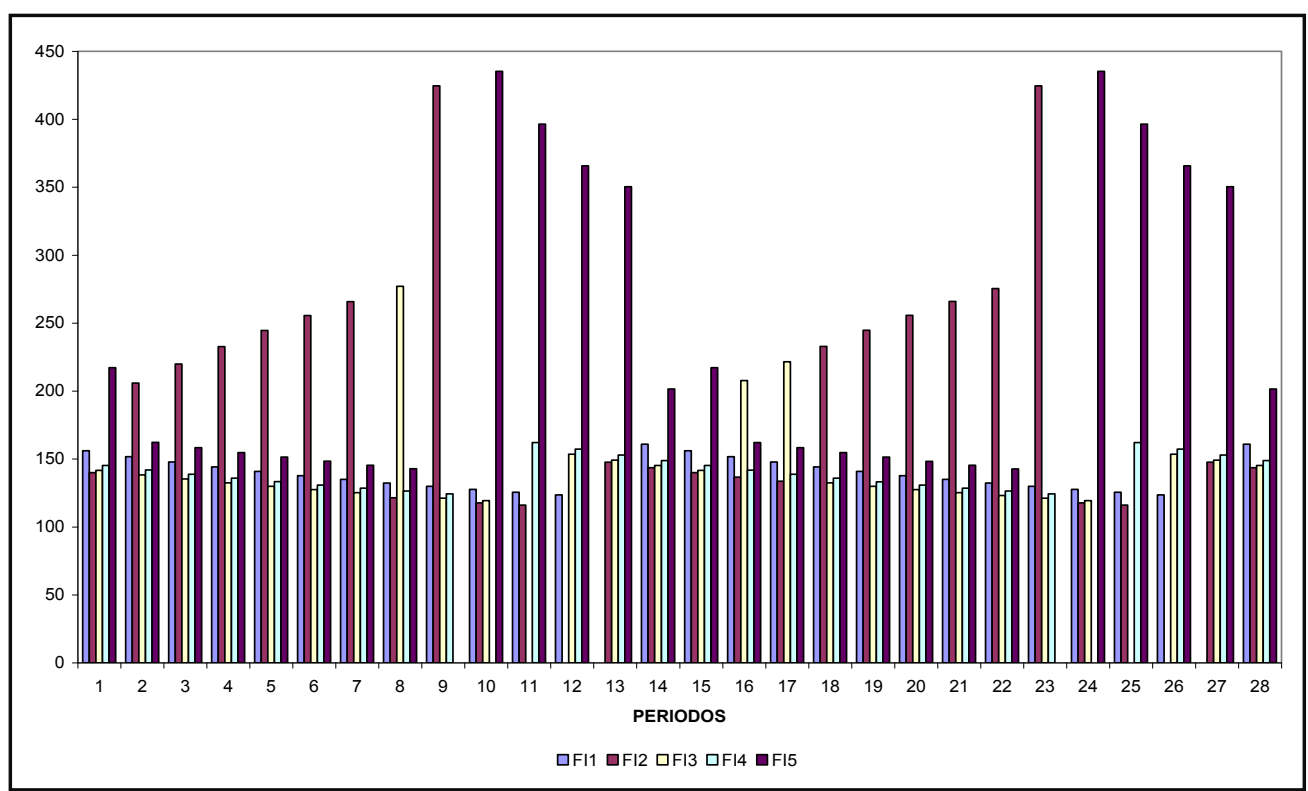

Figura 3 - Caudales $(\mathrm{t} / \mathrm{h})$ alimentados a las líneas en los diferentes períodos Fuente: Obtenidos del modelo propuesto

Los períodos en los que se deben detener las líneas de evaporación para realizar las tareas de limpieza, se muestran en la Tabla 2.

Tabela 2 - Períodos en los que se deben detener las líneas de evaporación para efectuar tareas de limpieza

\begin{tabular}{ccc}
\hline Línea (i) & $\mathbf{T P}_{\mathbf{1}, \mathbf{i}}$ & $\mathbf{T P}_{\mathbf{2}, \mathbf{i}}$ \\
\hline $\mathbf{1}$ & 13 & 27 \\
$\mathbf{2}$ & 12 & 26 \\
$\mathbf{3}$ & 11 & 25 \\
$\mathbf{4}$ & 10 & 24 \\
$\mathbf{5}$ & 9 & 23 \\
\hline
\end{tabular}

Fuente: Obtenidos del modelo propuesto 
Los perfiles de las resistencias globales para los evaporadores de la línea 1 se muestran en la Figura 4 mientras que los perfiles de concentraciones y de caudales a la salida de las unidades de evaporación de esa línea se muestran en las Figuras 5 y 6 respectivamente.

RESISTENGIA GLOBAL EN LINEA 1

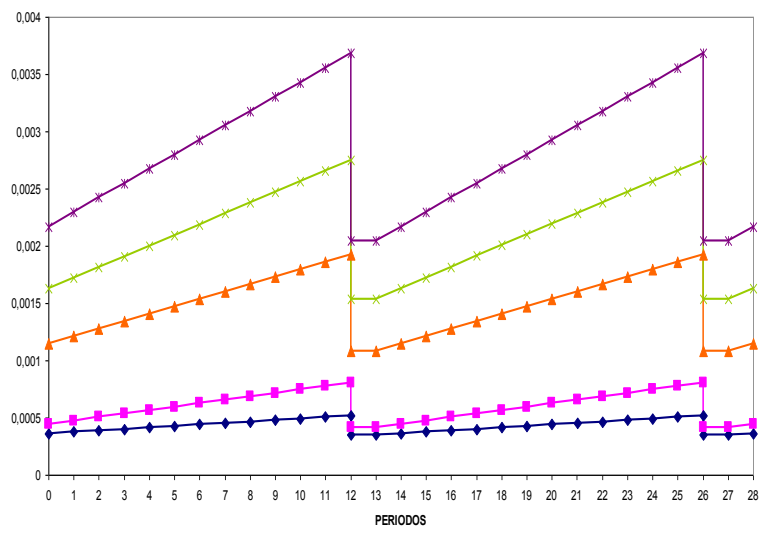

$\rightarrow \mathrm{R} 1,1 \rightarrow \mathrm{R} 1,2 \rightarrow \mathrm{R} 1,3 \rightarrow \mathrm{R} 1,4 \rightarrow \mathrm{R} 1,5$

Figura 4 - Perfil de las resistencias globales para las unidades de evaporación en línea 1

Fuente: Según el modelo lineal
CONCENTRACIONES EN LINEA 1

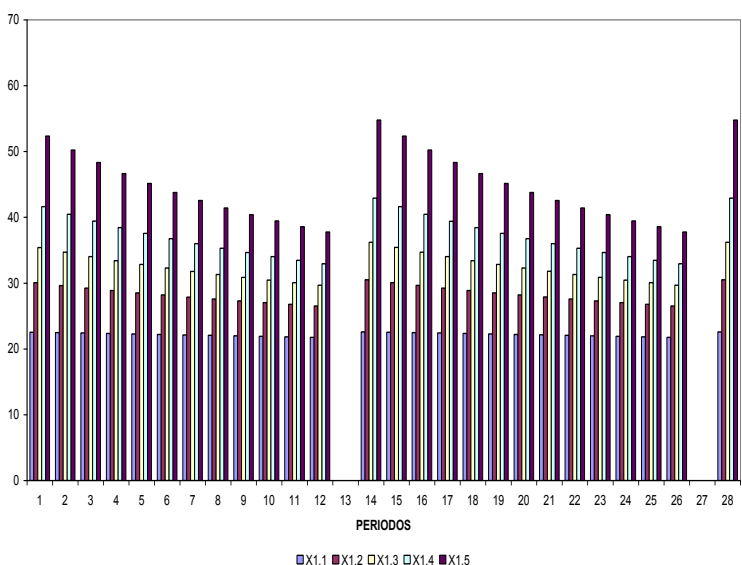

Figura 5 - Perfiles de concentraciones a la salida de las unidades de evaporación en línea 1 Fuente: Obtenidos del modelo propuesto

CAUDALES EN LINEA 1

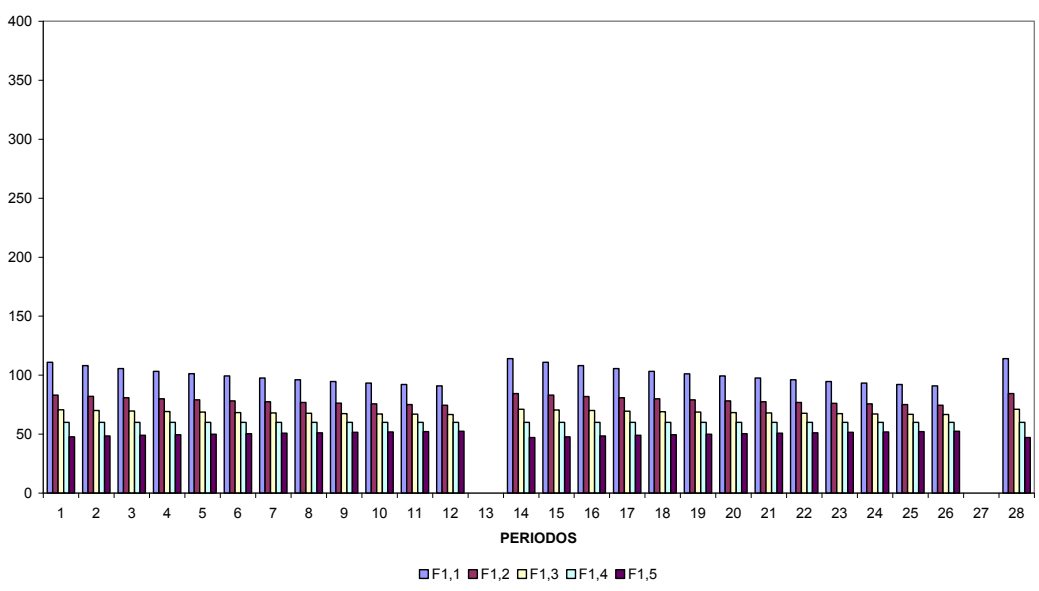

Figura 6 - Perfiles de caudales a la salida de las unidades de evaporación en línea 1 Fuente: Obtenidos del modelo propuesto

La implementación de este plan de operación requiere un sistema de control asociado que permita variar la alimentación a cada línea en los diferentes períodos, ya que como se observa en la Figura 3 dichos caudales varían en todos los períodos considerados.

Como se explicó oportunamente, en el modelo se exigen dos limpiezas en 14 días (28 períodos de 12 horas cada uno) para estar de acuerdo con la práctica usual en la industria azucarera de realizar una limpieza semanal a cada línea de evaporadores.

La solución indica, como se muestra en la Tabla 2, que las paradas para mantenimiento de las diferentes líneas de evaporación se deben realizar de manera consecutiva y dicha 
situación se repite para ambas limpiezas. Se debe comenzar con la línea 5 en el período 9 y finalizar con la línea 1 en el período 13 para la primera limpieza. La segunda limpieza comienza con la línea 5 en el período 23 y termina con la línea 1 en el período 27 (los períodos de parada para las líneas 2,3 y 4 se muestran en la Tabla 2). Todas las líneas operan durante 13 períodos antes de detenerse para realizar tareas de limpiezas y esta situación se verifica tanto para la primera como para la segunda limpieza.

Observando la evolución de las resistencias globales a la transferencia de calor, se nota que la menor resistencia se obtiene en el primer efecto de una línea y la mayor en el último. Esto es a que la viscosidad de los fluidos se incrementa por el aumento de la concentración. Como se puede ver en la Figura 3 el valor de la resistencia global en cada efecto para el período 1 es idéntico al del período 28 , lo que permite la operación cíclica de las líneas hasta obtener la producción deseada.

La línea 5 junto con la línea 2 son las que mayor caudal de jugo alimentado reciben en la mayoría de los períodos (ver Figura 3). Este comportamiento corresponde a un balance entre el área de intercambio y la pérdida de eficiencia por ensuciamiento de las líneas. En general la alimentación es similar en casi todos los períodos para las restantes líneas (numeradas como 1, 3 y 4).

Se observa que cuando se limpia la línea 5, el caudal alimentado a la línea 2 se incrementa abruptamente (períodos 9 y 23), mientras que los caudales alimentados a las líneas 1, 3 y 4 no experimentan variaciones abruptas. Esto implica que el caudal correspondiente a la línea 5 es derivado mayormente a la línea 2 durante las limpiezas de la primera. La alimentación para las líneas 1, 3 y 4 es en general regular sin variaciones abruptas, salvo para la línea 3 en el período 8 y en menor medida los períodos 16 y 17.

Se observa que cuando se detienen las líneas 1 a 4 para realizar operaciones de limpieza, casi todo el caudal correspondiente a dichas líneas es alimentado siempre a la línea 5.

A los efectos de comparar los resultados obtenidos por el modelo con la práctica usual en las plantas de azúcar, se calcularon las concentraciones alcanzadas a la salida de las líneas de evaporación en la siguiente situación: 4 líneas se limpian sucesivamente mientras que la quinta línea opera con los caudales que se derivan de las líneas en proceso de limpieza.

Cuando las 5 líneas se encuentran operando se alimenta $160 \mathrm{t} / \mathrm{h}$ a cada una con el objeto de procesar la totalidad del caudal $(800 \mathrm{t} / \mathrm{h})$. En los periodos en que operan 4 líneas y la restante está siendo limpiada, se alimenta a cada línea operativa 200 t/h de jugo. El ciclo dura 7 días (168 horas) y se repite mientras la planta está en producción. La función objetivo en 
este caso es de 4870. Comparando este resultado con el valor óptimo, 5717, se ve que este último es sensiblemente superior, con la consecuente disminución de los costos de energía.

\section{CONCLUSIONES}

Se abordó el problema de planeamiento de corto plazo de manera simultánea de líneas de evaporadores trabajando en paralelo mediante un modelo de programación no lineal mezcla entera. La formulación contempló la perdida de eficiencia de los equipos debido al ensuciamiento.

Los parámetros que reflejan el ensuciamiento fueron obtenidos de datos medidos en planta para el caso de estudio presentado. El modelo determina el programa de paradas para realizar tareas de mantenimiento/limpieza necesarias para recuperar la eficiencia de la transferencia de calor. Para cada período se obtienen los perfiles de caudal alimentado a cada línea y los correspondientes perfiles a la salida de caudales y concentraciones.

Como caso de estudio se tomó el sector de evaporación de un ingenio azucarero. La operación del sistema de evaporación optimizada, permite obtener una mayor concentración del producto de la evaporación con el consecuente ahorro de energía del arreglo evaporación/cristalización.

La principal fuente de incertidumbre del modelo proviene de la obtención de valores reales de variación de la resistencia global a la transferencia de calor con el tiempo, no solamente porque su determinación es complicada sino también por la falta de mediciones en los equipos de evaporación de los ingenios azucareros. Por otro lado, para implementar prácticamente el caso optimizado se necesita contar con un sistema de control de la planta que permita una distribución bastante precisa de los caudales alimentados a cada línea de evaporación en cada uno de los periodos considerados y los ingenios azucareros en general no poseen un sistema de control tan sofisticado en la evaporación lo que dificultaría la implementación en planta de los resultados óptimos.

Un trabajo interesante a realizar en el futuro es el análisis de sensibilidad de la función objetivo al modelo de variación de la resistencia global a transferencia de calor en el tiempo. 


\section{AGRADECIMIENTOS}

Los autores desean agradecer el financiamiento obtenido por "Consejo de Investigaciones de la Universidad Nacional de Tucumán”, "Consejo Nacional de Investigaciones Científicas y Técnicas de Argentina” y "Universidad Nacional del Sur".

\section{NOMENCLATURA}

\section{Parámetros y variables}

C1, C2 Parámetros del modelo resistencia global de transferencia de calor

$F \quad$ Caudal a la salida de un evaporador $(\mathrm{t} / \mathrm{h})$

FI Caudal alimentado a una línea de evaporación $(\mathrm{t} / \mathrm{h})$

F0 Flujo total alimentado al sistema $(\mathrm{t} / \mathrm{h})$

FO Función objectivo

M Número de unidades en una línea

$N \quad$ Número líneas en paralelo

$R \quad$ Resistencia global a la transferencia de calor $\left(\mathrm{h} \mathrm{m}^{2}{ }^{\circ} \mathrm{C} / \mathrm{kcal}\right)$

TC Período de parada

$V \quad$ Flujo de vapor $(\mathrm{t} / \mathrm{h})$

$x \quad$ Concentración (\%)

$x_{M} \quad$ Concentración a la salida de la evaporación (\%)

$x_{0} \quad$ Concentración de la alimentación (\%)

\section{Indices}

$h \quad$ Parada por limpieza

$i \quad$ Línea

$j \quad$ Posición en una línea

$t \quad$ Período

\section{Variables Binarias}

$a i, t, b i, t \quad$ Variables auxiliares para definer a $\mathrm{z}$

$y m, i, j \quad$ Indica si una línea está parada

$z h, i, t \quad$ Indica ciclo de limpieza 


\title{
OPERATIONAL SCHEDULING OF EVAPORATION NETWORKS
}

\begin{abstract}
In several industrial processes, evaporation is a key operation in order to an efficient water and energy management. In this paper, the scheduling of multiple effect evaporation systems is addressed. The objective of this work is to determine the optimal shutdown sequence of the evaporation lines to perform maintenance/cleaning tasks. A mixed integer nonlinear programming model (MINLP) based on a discrete time representation is presented where equipment performance decay is considered. The model is applied to the evaporation system of a cane sugar plant. Optimal shutdown sequence, operating time for each evaporation line, concentration and fed flow profiles are obtained as results. The optimization of the case study allows obtaining a higher product concentration at the evaporation system, which implies energy savings within the evaporation/crystallization system.
\end{abstract}

Keywords: Evaporation. Operational scheduling. Mathematical programming. Cane sugar.

\section{REFERENCIAS}

GEORGIADIS, M.C.; PAPAGEORGIOU, L.G., MACCHIETTO, S. Optimal cyclic cleaning scheduling in heat exchanger networks under fouling. Computers \& Chemical Engineering, v. 23, Supplement, p. S203-S206, European Symposium on Computer Aided Process Engineering, Proceedings of the European Symposium, 1999.

GEORGIADIS, M.C.; PAPAGEORGIOU, L. G. Optimal energy and cleaning management in heat exchanger networks under fouling. Chemical Engineering Research and Design, v. 78, n. 2, p. 168-179, 2000.

HELUANE, H. et al. Scheduling of continuous parallel lines in the evaporation section of sugar plants. Chemical Engineering Communications, v. 191, n. 9, p. 1121-1146, 2004.

HELUANE, H. et al. Enhancing sugar cane process performance through optimal production scheduling. Chemical Engineering and Processing: Process Intensification, v. 46, n. 3, p. 198-209, 2007.

JAIN, V.; GROSSMANN, I.E. Cyclic scheduling of continuous parallel process units with decaying performance. AIChE Journal, v. 44, n. 7, p. 1623-1636, 1998.

HOUZE, M.; JUHASZ, N.; GROSSMANN, I.E. Optimization model for production and scheduling of catalyst replacement in a process with decaying performance. In: Proceedings FOCAPO, 2003.

SCHULZ, E.P.; BANDONI, J.A.; DIAZ M. S. Optimal shutdown policy for maintenance of cracking furnaces in ethylene plants. Industrial \& Engineering Chemistry Research, v. 45, n. 8 , p. $2748-2757,2006$.

HELUANE, H. Optimización de la operación de unidades continuas en paralelo en plantas Agroindustriales. Tesis Doctoral Facultad de Ciencias Exactas y Tecnologías Universidad Nacional de Tucumán (Argentina). 
GAMS. A Users' Guide. GAMS Development Corporation, 2008a.

GAMS. The Solvers Manual. GAMS Development Corporation, 2008b.

Originais recebidos em: 17/09/2012

Aceito para publicação em: 19/07/2013

Iberoamerican Journal of Industrial Engineering, Florianópolis, SC, Brasil, v. 5, n. 9, p. 358-375, 2013. 375 